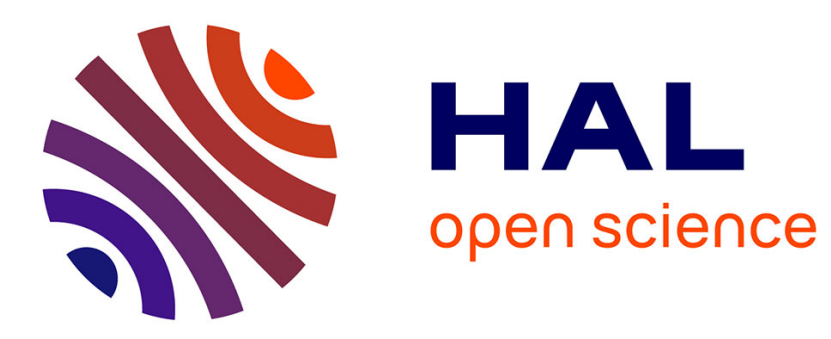

\title{
Perimeter Control as an Alternative to Dedicated Bus Lanes - A Case Study.
}

Nicolas Chiabaut, Michael Küng, Monica Menendez, Ludovic Leclercq

\section{To cite this version:}

Nicolas Chiabaut, Michael Küng, Monica Menendez, Ludovic Leclercq. Perimeter Control as an Alternative to Dedicated Bus Lanes - A Case Study.. TRB 2018, Transportation research board annual meeting, Jan 2018, Washington DC, United States. 18p. hal-02004367

\section{HAL Id: hal-02004367 \\ https://hal.science/hal-02004367}

Submitted on 27 May 2021

HAL is a multi-disciplinary open access archive for the deposit and dissemination of scientific research documents, whether they are published or not. The documents may come from teaching and research institutions in France or abroad, or from public or private research centers.
L'archive ouverte pluridisciplinaire HAL, est destinée au dépôt et à la diffusion de documents scientifiques de niveau recherche, publiés ou non, émanant des établissements d'enseignement et de recherche français ou étrangers, des laboratoires publics ou privés. 


\title{
Perimeter Control as an Alternative to Dedicated Bus Lanes - A Case Study
}

\author{
Nicolas Chiabaut * \\ Univ. Lyon, ENTPE / IFSTTAR, LICIT \\ UMR_T 9401, F-69518, LYON, France \\ Tel: +33472047758 \\ Email: nicolas.chiabaut@entpe.fr \\ Michael Küng \\ ETH Zurich \\ Stefano-Franscini-Platz 5, CH-8093, Zurich, Switzerland \\ 1 Tel: +41797680336 \\ 12 Email: mikueng@student.ethz.ch \\ 13 Monica Menendez \\ 14 ETH Zurich \\ 15 Stefano-Franscini-Platz 5, CH-8093, Zurich, Switzerland \\ 16 Tel: +41446336695 \\ 17 Email: monica.menendez@ivt.baug.ethz.ch

\section{Ludovic Leclercq} \\ Univ. Lyon, ENTPE / IFSTTAR, LICIT \\ UMR_T 9401, F-69518, LYON, France \\ Tel: +33472047716 \\ Email: ludovic.leclercq@entpe.fr \\ * Corresponding author
}

\section{Paper 18-00029}

Paper submitted for presentation and publication at the $97^{\text {th }}$ Annual Meeting Transportation Research Board, Washington D.C., January 2018

Special call for paper "Multimodal system analysis and modeling" AHB45 - Traffic Flow Theory and Characteristics Committee

Word count: 5424 words +4 figure $(s) \times 250+2$ table $(s) \times 250=6924$ words 
1 ABSTRACT

2 Dedicated bus lanes (DBL) are a common traffic management strategy in cities all over the world, 3 as they improve the efficiency of the transit system clearly by preventing buses from getting trapped 4 in traffic jams. Nevertheless, DBL also have certain disadvantages. In particular, they consume space, reduce available capacity for general traffic, and can thus lead to even more congested car traffic situations. Hence, it is appealing to find more efficient alternatives that maintain a sufficient network supply for general traffic while guaranteeing high commercial speeds for the bus system. To this end, this paper investigates if perimeter control (gating) can be such an alternative to DBL strategies. This solution aims at controlling the traffic conditions of a given area by monitoring 


\section{INTRODUCTION}

Traffic congestion and its negative impacts is an ongoing topic of discussion among city authorities all over the world, and will continue to remain on the agenda as urban economic development (1) and number of vehicles are increasing (2). Some regions (e.g. city centers) are predestined for bottlenecks due to its function as junctions and economic hubs. Once congestion arises in these sensitive zones, congested traffic states start to propagate in the surrounding network sections and may lead to major gridlocks. Therefore, it becomes crucial to monitor and control in real-time urban traffic conditions in order to utilize the existing network as efficiently as possible (3) and minimize congestion hours in such critical regions.

Urban congestion has direct effects on both travelers (increased travel time, reduced speed, decrease in time reliability, greater fuel consumption, higher wear, etc.) and the environment (increased pollution and noise). In addition, traffic jams affect also other transportation modes, including public transport. In particular, bus lines might experience increases in both, average travel times and unreliability for passengers (4). As a result, the transit system looses appeal and the shift of transportation mode from individual vehicles to massive transportation modes as emphasized in (5) can decrease. Local authorities and operators are therefore compelled to deploy strategies to improve performance and increase attractiveness of the public transport system, paying special attention to bus operations.

A common measure deployed worldwide to reduce bus delays is the use of dedicated bus lanes (DBLs), which protect bus operation from general traffic by reserving a special lane. Among others, (6) and (7) emphasize various advantages of DBL, in particular the fact that they prevent buses from getting trapped in traffic queues at signalized intersections, significantly improving thereby the bus system performance. Nevertheless DBLs still remain controversial and not always well accepted. Especially, (8) points to the unavailability of the dedicated lane for individual vehicles as a main drawback, whereby capacity for cars is decreased. As a consequence DBL are only appropriate for low traffic flows from a car user perspective $(9,10)$, a disadvantage that must be taken into account. Research is ongoing in the field of DBLs. Pre-signals, as proposed by (11), can be used to better use the intersection capacity even when bus flows are low; hence keeping the bus priority and reducing the negative effects on cars. Intermittent bus-lanes, as proposed by (12), are a further development. Thereby, DBLs are opened intermittently to general traffic in order to mitigate the negative effects of DBLs on car traffic while keeping bus operations optimal. However, although IBL have been deeply analytically studied $(8,9)$, tentatives of real-world deployment remain very rare. On a larger scale, decisions for DBL deployment can also serve different purposes like promoting the use of public transport by reducing space for cars, which makes it a matter of political intentions.

The current paper proposes an alternative to DBL by applying the concept of Perimeter Control, also known as Gating, to a sensitive region and replacing at the same time the bus lanes in the area with general purpose lanes. More precisely, this approach is tested on an urban arterial. Perimeter control as described by (13) addresses the mentioned point of increasing traffic efficiency in a existing network. Basically, the idea of this control strategy is to restrict the input flow to a predefined area, in order to maintain non-congested traffic states inside that region (14) and improve thereby the overall traffic performance. Thus, congestion is relieved on the network level. More exactly, the incoming flow is controlled through the traffic signals at the boundaries of the "protected" area. One advantage of this approach is, that it can be implemented in real time through real traffic data. There are various examples of perimeter control strategies in literature 

with positive results, i.a. $(13,14,15,16,17,18,19,20)$. The strategy is successful when the saved delays in the controlled region are higher than the additionally generated delays by the controller at the entries. Perimeter control schemes are already applied in some cities. Zurich (19) or Chania (3) can be mentioned as real examples. A main drawback of gating is that long queues can occur at the perimeter entries, which lead possibly to spillbacks that affect the surrounding network (for details see $(14,16))$. Only a few papers focus more deeply on this issue. One approach can be found in (20), where the queue length at the perimeter boundary are considered and controlled in the model. (21) recently developed a so called multi-scale controller which takes the queue-lengths at perimeter entries into account by integrating the occurring delay in the queues directly into the objective function. Traffic performance is therefore improved globally by considering both the network scale and the local level.

If a high level of service can be obtained through perimeter control, the original purpose of DBL to guarantee free flow conditions for buses, becomes possibly redundant and DBL could be eliminated. This hypothesis is examined in the following by applying a multi-scale feedback perimeter controller as an alternative to DBL in a simplified simulation case study. In contrast to former studies, a multi lane urban arterial is considered as the controlled perimeter instead of a region. Similar to a city center, an arterial is often a traffic hot spot and a critical section for congestion emergence. The control scheme is tested in particular in the context to bus operations, as the travel times of buses are compared for cases with perimeter control and with DBL. Because of the worldwide presence of DBLs, the current case study is of relevance and can provide decision makers with new aspects, all the more since it strikes a new path by the complete replacement of DBL in contrast to the optimization of DBL examined in former research.

The paper is organized as follows. After describing the simulation and controller framework in Section 2, findings are presented in Section 3. This section starts by an evaluation of the controller to validate that the framework developed for large-scale traffic flow models works with a detailed description of traffic dynamics, i.e. in micro-simulation. Then, the controller can be safely applied to study the opportunity of replacing a DBL by a gating strategy. Section 4 is devoted to the conclusions.

\section{METHODOLOGY}

The concept for the multi-scale control approach applied in this work originates from (21), but the original methodology has been adapted to address different research questions and objectives. Especially, this method is adapted to a finest level of details, i.e. micro-simulation, whereas it has been originally developed for large-scale models as the biggest part of existing papers. (21) provides the accurate framework to implement and test in simulation a multi-scale control that aims at maintaining a protected area in free-flow conditions while simultaneously limiting spillbacks at the entries of the protected area. Different scenarios, presented in the next section, will be simulated.

\section{Case Study}

All the simulations for the present work were executed with the traffic simulation platform SymuVia. SymuVia is a dynamic microscopic traffic simulator and provides thus a very high level of detail. It is based on the kinematic wave model (KW) described by (22) as well as (23) with a Lagrangian resolution introduced in (24). Multiples components to reproduce urban networks (intersections, lane-changing, multi-class, etc.) are embedded in the simulator and the positions of all 
vehicles are calculated every second giving access to their full trajectories. Furthermore, SymuVia

\section{Network}

Figure 1 gives an overview of the network-set up and of the controlled intersections in the case study. The network consists of a 14 x 14 street grid, also known as a Manhattan-type network. This layout is large enough to represent roughly a district of a city with its interlinked traffic dynamics. In the center of the network, an arterial road crosses the whole district. All links are 200 meters long. The arterial has three lanes and is one-directional from west to east. The rest of the links are bi-directional with one lane in each direction. The speed limit for the arterial is 50 $\mathrm{km} / \mathrm{h}$, and $30 \mathrm{~km} / \mathrm{h}$ for the rest of the links.

Vehicles can enter and leave the arterial at any of its intersections. Only vehicles on the very left lane can turn into a side-street on the left hand side and same holds equally for right-turning vehicles. Vehicles entering the arterial from the side-streets have to take the nearest lane (i.e. most left or most right lane). Once cars move on the arterial, they can switch lanes without restriction.

Two regions are denoted: the arterial is considered as a center region $A$, whose traffic states should be controlled, the side streets represent the outer region $B$. On every intersection the total cycle length of the signals is 60 seconds, and there is neither intermediate time nor a yellow phase. Each cycle contains two phases, where the two opposite approaches belong to the first phase and the arterial to the second. On side street intersections, green time is split evenly $30 \mathrm{~s} / 30 \mathrm{~s}$ for both phases. For arterial-side-street intersections, which are controlled later, the arterial gets assigned $40 s$ of green time and the side street entries $20 s$ of green time for the cases without control. Due to the higher demand, the arterial has also a higher green-time ratio, which is assumed to be realistic and consistent with the hierarchy of real urban networks. In total there are 14 intersections linking the arterial with the outside region, on which the controller is applied via the traffic signals, i.e. green times are updated at each control period. 
(a)

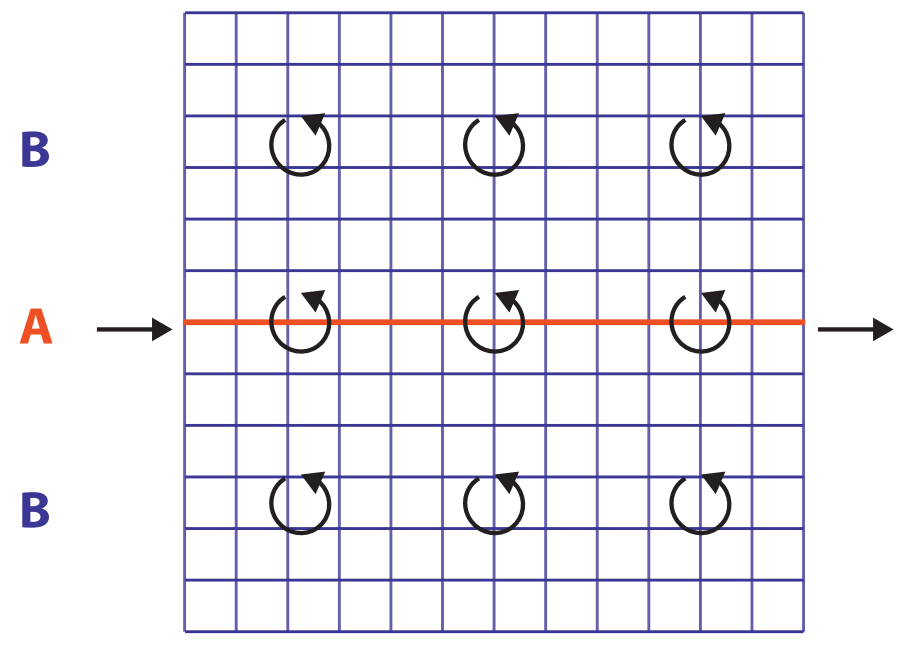

(b)

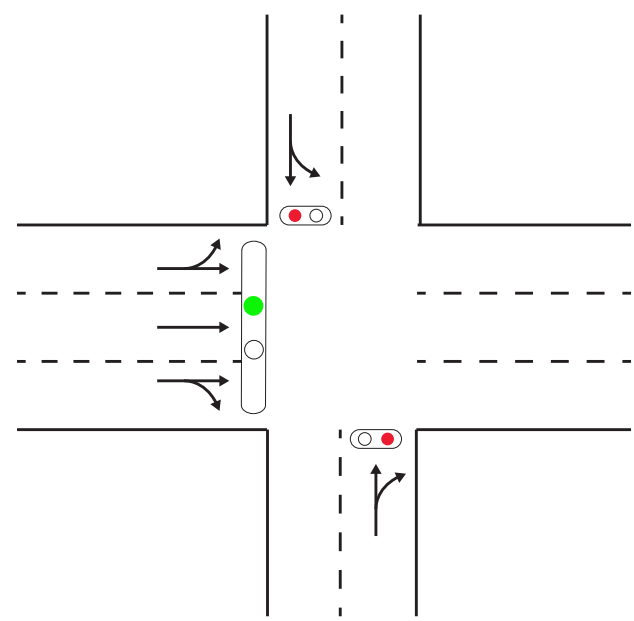

FIGURE 1 (a) Manhattan-type network with an arterial as the controlled region A and the outer region B. Arrows represent inner and outer demand. (b) Schematic intersection arterial - side street with controlled traffic signals and traffic streams

\section{Demand}

There are two types of demand. First, there is an outer demand entering and leaving the network through the arterial and passing the network from left to right. Second, there is an inner demand with both, origin and destination within the network. To generate this demand, the network is divided into nine zones of equal size and a demand-level is defined for the number of vehicles traveling from each zone to each other zone. The arterial (outer) demand is fixed to $2600 \mathrm{veh} / \mathrm{h}$, while the zone-to-zone (inner) demand varies over time with the intention to simulate a typical peak period - $300 \mathrm{veh} / \mathrm{h}$ during the first 30 minutes, $600 \mathrm{veh} / \mathrm{h}$ during the second 30 minutes, and 300 again veh/h during the last 30 minutes. A simulation run lasts 90 minutes.

\section{Multi-Scale Feedback Control Approach}

The controller is feedback-based, which means that the control action is dependent on the previous output. It is worth noting that the proposed methodology can be used with other control schemes. Traffic detectors (sensors) measure indirectly the control variable $J_{D}$ (total time spent) while the traffic signals parameters (actuator) influence the control variable (green time ratios for every perimeter intersection). The detectors are placed on every street entering and leaving the arterial. As mentioned, the challenge of the multi-scale control algorithm are the two competing control objectives, which need to be coupled into an integrated control scheme (21). The traffic performance should be optimized simultaneously at the network and the local level. As indicator for the global traffic state in the protected perimeter we use the total vehicle accumulation in region $A$. For the local level, the total delay, which is directly related to the queue lengths, at the entry intersections are considered as indicators. Traffic conditions are monitored through virtual detectors which give access to the total vehicle accumulation of the zone as well as to queue lengths at the entries. Notice that accumulation is directly connected to the total time spent by vehicles inside the arterial. The applied controller calculates the optimal green time ratios for every entry 
intersection, based on the measured data, in order to optimize the two objectives mentioned above. The new green time ratios are updated and the traffic signal parameters are specifically modified at each intersection every $K$ seconds. This implies that one control period lasts $K$ seconds. To change signal timing more often seems not realistic in this context as cycle length on intersections is 60 seconds for our case study.

The optimization problem is formulated and explained by the following equations, where (1) is the objective function and equations (2) - (10) are the constraints. Note that the dependence of variables to the time period may be omitted in the following formulation to make the writing easier.

$$
\min J_{D}=\sum_{p=1}^{L}\left(K *\left(n_{A A}+n_{A B}\right)+\sum_{i \in I} \sum_{m \in M \backslash M_{\text {out }}^{i}} x_{m}^{i} * k_{x} * \frac{c-g_{m}^{i}}{2} * \frac{K}{C}\right)
$$

s.t.

$$
\begin{aligned}
n_{A A}(k+1) & =n_{A A}(k)+\lambda_{\text {Arterial } 0} \cdot K-\lambda_{\text {Arterial } 14} \cdot K \\
n_{A B}(k+1) & =n_{A B}(k)+\lambda_{B A} \cdot K-\lambda_{A B} \cdot K \\
\lambda_{B A} & =\sum_{m \in M \backslash M_{\text {out }}^{i}} \mu_{m}^{i} \\
\lambda_{A B} & =\sum_{m \in M \backslash M_{\text {in }}^{i}} \mu_{m}^{i} \\
\mu_{m}^{i} & =\min \left\{q_{m}^{i},\left(s_{m}^{i} * g_{p}^{i}\right)\right\} \\
x_{m}^{i} & =\frac{u * w}{u+w} *\left(c-g_{m}^{i}\right) * \frac{q_{m}^{i}}{\left(q_{\max }-q_{m}^{i}\right)} \\
q_{\max } & =\frac{u * w}{u+w} * k_{x} \\
g_{p, \min }^{i} & \leq g_{p}^{i} \\
g_{p, \max }^{i} & \geq g_{p}^{i}
\end{aligned}
$$

In the objective function (1), the total travel costs $J_{D}$ are minimized. These travel costs are composed of (i) the total time spent by vehicles in region $A$ (arterial) during one control period, i.e. the first two terms multiplied by the length $K$ of the control period, and (ii) the total delay at the different entry intersections. According to KW theory, this delay is calculated as the product of the queue length $x_{m}^{i}$ with $k_{x} * \frac{c-g_{m}^{i}}{2} * \frac{K}{C}$ where $k_{x}$ is the jam density, $g_{m}^{i}$ is the green time for stream $m$ at intersection $i, c$ is the cycle duration and $\frac{K}{C}$ is the number of traffic signal cycles during one control period. The queue lengths in outflowing direction are not taken into consideration here, as they are accounted for when computing the accumulations in the protected region. Note that the two objective criteria have the same weight, which can be adjusted for different purposes, according to the corresponding main aim, see also (25). Constraints (2) and (3) stand for the vehicle accumulation in region $A$, which is basically inflow minus outflow. The predefined flow entering and leaving region $A$ through the arterial is simple to measure with loop detectors. Meanwhile the flow that enters and exits of region $A$ through the side streets is the sum over all intersections ((4) and (5)). This flow must be the minimum of the measured demand on the upstream loop detectors 
and the capacity of the entry (6)). The capacity however depends on the allocated green time for the entry and changes therefore with every control cycle. Equation (7) shows how mean queue lengths during one period are estimated based on $\mathrm{KW}$ model, taking into account the measured arrival flow, the green time and the link capacity (8). Contrary to (? ), we decide to express the length of the queue in terms of distance. Because the controller is based on a detailed description of traffic dynamics, it makes it possible to precisely track the propagation of the queue. To this end, virtual loop detectors have been emulated at the entry of the approach links, giving access to the average demand $q_{m}^{i}$ during one control period. This value that is then used to derive the average length of the queue for the control period. If the queue cannot disappear during the control period, equation (7) will underestimate the length of the queue until the queue spills back on the detector located at the entry of the approach link. Consequently, queue lengths are approximated during transition phase. Moreover, it is important to notice that, in the case of strong spillbacks, equation (7) strongly underestimates the length of the queues and the associated delays in the objective function (1). However, in such a case, a DTA process will be required because congestions spreading on region $B$ will have an impact on route choices and traffic assignments. Consequently, the controller propose here is valid only when the queues can be maintained within the approach links. Validated by visual inspection of the simulated results, the assumption seems appropriate and this analytical formulation gives an accurate average estimation of the queues' lengths. Furthermore a minimum and maximum allowed green time-ratio for the entries is defined by (9) and (10). These boundaries were defined to emulate realistic scenarios. It seems not plausible to have green durations below six seconds. Same holds for the maximum boundary. If the red sequence for the high-demand arterial becomes too long, this leads immediately to spillbacks. Note that, for an overview, table 1 lists the used variables. 
TABLE 1 Nomenclature

\begin{tabular}{|c|c|c|}
\hline Variable & Description & Value (if constant) \\
\hline$J_{D}$ & Total travel costs & \\
\hline$K$ & Control period $[s]$ & 300 \\
\hline$k$ & Index of the current control period & \\
\hline$n_{a b(k)}$ & $\begin{array}{l}\text { Calculated accumulation of vehicles in Region } a \text { with } \\
\text { destination in Region } b \text { at the end of period } k, a, b=A, B \text { [veh] }\end{array}$ & \\
\hline$I$ & Set of intersections & \\
\hline$L$ & Prediction horizon [number of control period] & 18 \\
\hline$P^{i}$ & Set of phases of intersection $i, i \in I$ & \\
\hline$M^{i}$ & Set of streams an intersection $i, i \in I$ & \\
\hline$x_{m}^{i}(k)$ & $\begin{array}{l}\text { Calculated mean queue length of stream } m \text { at intersection } i \\
\text { during period } \mathrm{k}, \mathrm{i} \in I, \mathrm{~m} \in M \text { [veh] }\end{array}$ & \\
\hline$\lambda_{\text {Arterial } i}$ & $\begin{array}{l}\text { Predefined arterial flow entering respectively measured } \\
\text { arterial flow leaving the network at intersection } i=0,14 \text { [veh } / \mathrm{h}]\end{array}$ & 2600 (inflow) \\
\hline$\lambda_{a b}$ & Calculated total flow from region $a$ into region $b, a, b=A, B$ [veh $/ \mathrm{h}]$ & \\
\hline$\mu_{m}^{i}$ & $\begin{array}{l}\text { Calculated departure flow of stream } m \\
\text { at intersection } i \text { in period } k \text {, } \\
i \in I, \mathrm{~m} \in M^{i}[\mathrm{veh} / \mathrm{h}]\end{array}$ & \\
\hline$q_{m}^{i}$ & $\begin{array}{l}\text { Measured arrival flow of stream } m \\
\text { at intersection } i \text { in period } k, i \in I, m \in M^{i}[\mathrm{veh} / \mathrm{h}]\end{array}$ & \\
\hline$s_{m}^{i}$ & $\begin{array}{l}\text { Maximum discharging flow of stream } m \\
\text { at intersection } i \text { during green in period } k, i \in I, m \in M^{i}[\mathrm{veh} / \mathrm{h}]\end{array}$ & \\
\hline$g_{p}^{i}$ & $\begin{array}{l}\text { Green time ratio of phase } p \text { at intersection } i \text { for cycle } C \\
\text { during period } p, i \in I, p \in P^{i}\end{array}$ & \\
\hline$u$ & Free flow speed on a link corresponding to the speed limit $[\mathrm{m} / \mathrm{s}]$ & $13.89(\mathrm{~A}) ; 8.34(\mathrm{~B})$ \\
\hline$w$ & Shock wave speed of vehicles $[\mathrm{m} / \mathrm{s}]$ & -5.8 \\
\hline$r$ & Red time of corresponding side street approach & \\
\hline$q_{\max }$ & Capacity-flow of one lane [veh/h] & 2200(A);1860(B) \\
\hline$k_{\max }$ & Jam density of one lane [veh/m] & 0.15 \\
\hline$g_{p, \min }^{i}$ & $\begin{array}{l}\text { Minimum allowed green time ratio for phase } p \\
\text { at intersection } i, i \in I, p \in P^{i}\end{array}$ & 0.1 \\
\hline$g_{p, \max }^{i}$ & $\begin{array}{l}\text { Maximum allowed green time ratio for phase } p \\
\text { at intersection } i, i \in I, p \in P^{i}\end{array}$ & 0.67 \\
\hline
\end{tabular}

1 Input Data

2 As it is evident from the objective equation (1), the required data inputs for the controller are: traffic accumulation in region $A$ and queue lengths at entry intersections. The output are the green ratios, which are then used in the computations for the next period. For calculating other values, 
real-time simulated measurements are used. In order to obtain all the necessary data, a very dense virtual detector covering is required. Namely, detectors are placed on every entry and exit of the arterial. Approximation through extrapolation would be a possibility in case of a lack of detectors. Alternatively, different data sources or other types of detectors (e.g. microwave radar, video image detection) could serve as input. Besides that, connected vehicles as suggested by (21), GPS data from mobile phones (26), navigation devices (27), or a combination of sources, could provide the required input data too.

\section{RESULTS}

In the following, simulation results are interpreted and discussed. As previously explained, efficiency of the controller is first evaluated for the no DBL case. The objective is to validate that the framework developed for large-scale traffic flow models can be adapted to micro-simulation and is a consistent for a detailed description of traffic dynamics. This approach is complementary to the work of (21). Then, the controller can be safely applied to bus operations. Especially, we study the opportunity of replacing a DBL by a gating strategy while maintaining high commercial speed for the bus lines. To this end, four different scenarios can be identified, see 2. Obviously, comparison of Sc.2 with Sc.3 is the most insightful approach to examine if gating is an alternative of DBL. In addition, Sc. 4 will be used to evaluate the performance of the controller as mentioned earlier. Finally, Sc. 1 is set aside because it is probably too extreme and would not make sense to apply it in the real world.

TABLE 2 Scenarios

\begin{tabular}{|c|c|c|c|}
\multicolumn{3}{c|}{} & \multicolumn{3}{|c|}{ Dedicated Bus lane } \\
\hline \hline \multirow{3}{*}{ Controller } & & Yes & No \\
\cline { 2 - 4 } & Yes & 1 & 2 \\
& No & 3 & 4 \\
\hline \hline
\end{tabular}

\section{Efficiency of the Multi-Scale control scheme}

Primarily, the consequences of applying the presented controller to the urban arterial are illustrated by comparing $S c 2$ with $S c 4$. Traffic in the arterial is expected to move faster thanks to the control scheme which optimizes the signal plans.

\section{Traffic Conditions}

Here we define two variables as performance indicators: vehicle accumulation, mean speed, and mean density in the arterial. These parameters are common as traffic performance indicators, especially against the background of the MFD concept (28). Previous studies, such as (16) or (21), introduced the same measures. The results are described in Figure 2 below. Left column holds for the traffic performance in case $S c 4$ in contrast to case $S c 2$ on the right side. 

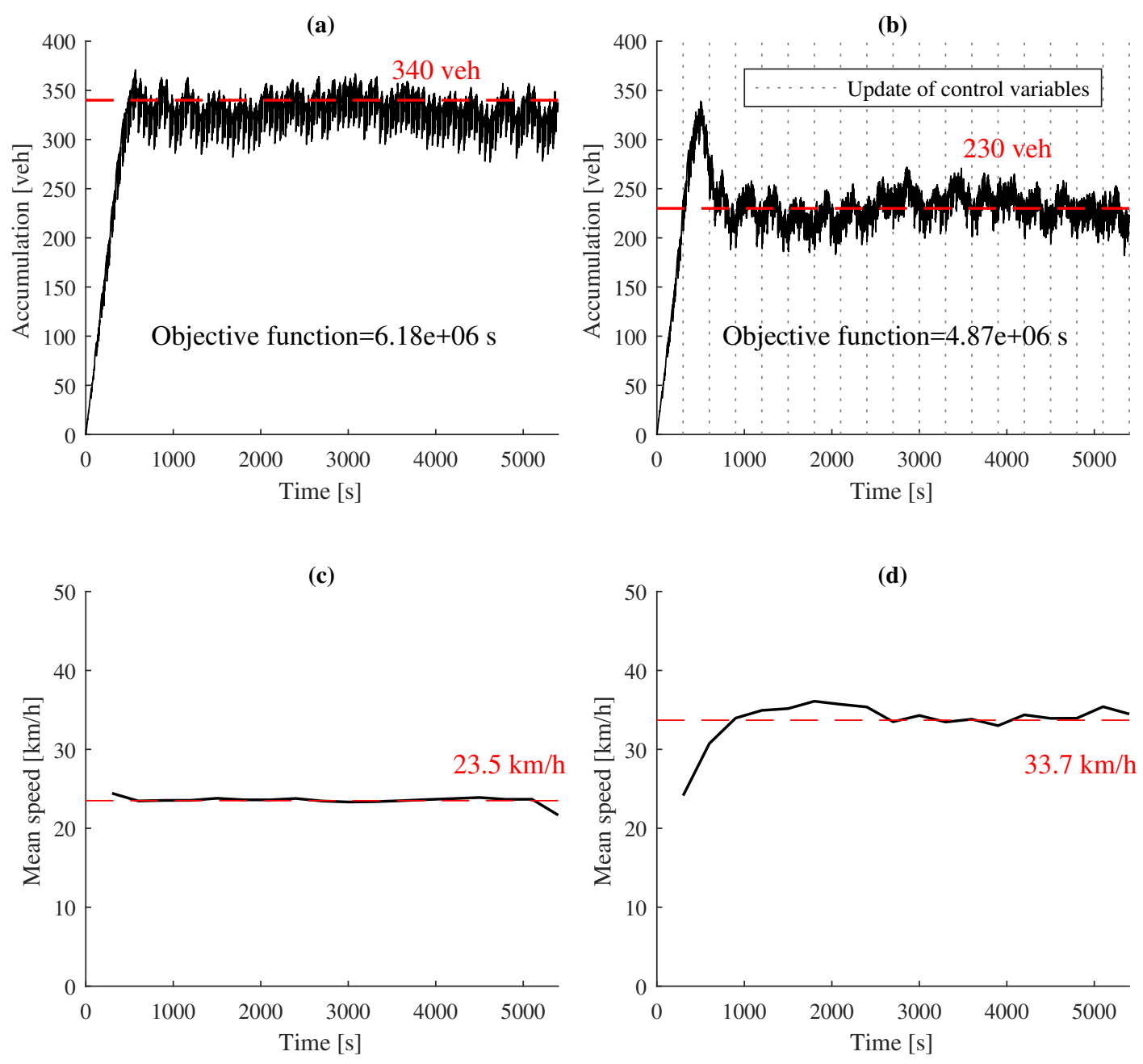

FIGURE 2 Traffic performance in the urban arterial illustrated by vehicle accumulation $(a, b)$, mean speed (c,d) and mean density (e,f). Left column: Case A. Right column: Case B

As expected under constant demand, the vehicle accumulation in the arterial stabilizes over time, although on a lower level when control scheme is implemented, see Figure 2a and b. This is because the controller extends green phases for the arterial if there is no or a small queue at the related entry. In average there are approximatively 100 vehicles less in the arterial for case B. The accumulation curve in Figure $2 \mathrm{~b}$ peaks at the beginning as new signal plans are allocated to the traffic lights for the first time after five minutes. Notice that the objective function is lower for $S c 2$ than $S c 4$. As a consequence the level drops again, when the controller starts operating. The controller interventions are illustrated in Figure $2 b$ through the vertical, dashed lines. Accordingly to less waiting time respectively less accumulation in the controlled arterial, vehicles can drive on a significantly higher mean speed, see Figure $2 \mathrm{c}$ and d. The difference between case $S c 2$ to case $S c 4$ is $10 \mathrm{~km} / \mathrm{h}$ in average. Overall, it can be stated, that the proposed controller improves traffic performance, represented by the three parameters named above, strongly and constant over the 
1 whole simulated period. Differences become even clearer when demand in the arterial is increased up to $3000 \mathrm{veh} / \mathrm{h}$ and higher. But in this case, heavy congestion is caused in the arterial for $S c 4$ and results become less illustrative for the comparisons. Another indicator for improved traffic performance is the total arterial output, both at the main arterial exit and at side street junctions. During the whole simulation run, nearly 300 vehicles more passing the arterial are measured in $S c 2$ compared to case $S c 4$.

\section{Application for bus operation}

In urban arteries or boulevards, DBLs are common in many cities as they promise various advantages as described for example by (29). Primarily bus times and service reliability are improved through DBLs. As shown in the previous section, the proposed controller enhances traffic performance in the arterial considerably, as a consequence traffic moves faster. From this point of view, one can argue, that the DBL becomes superfluous as their original purpose is now redundant. In addition, the DBL have the disadvantage of reducing the arterial capacity devoted to general traffic (8). When searching at optimizing the transportation system at a global level, such a strategy can be counter productive (10) Therefore, the potential DBL replacement by the perimeter control scheme is tested in the following by comparing the travel time of buses in the arterial for three cases and in the described simulation environment.

As previously explained, we now focus on the comparison of $S c 2$ with $S c 3$ where a DBL is deployed on the most right lane. Buses run with a frequency of 5 minutes through the arterial. There are five stations and dwell times are fixed to ten seconds for each station. These conditions hold for all three cases. Note that dynamic dwell times, i.e. dwell times that depend on the effective time headways between two successive buses, have been tested. Such an approach leads to similar results since bus system is very regular for the different test cases. Figure 3 provides the graphical results of the comparisons. The travel time of the two last buses in the simulation are neglected, because simulation stops before these buses arrive to their destination. 


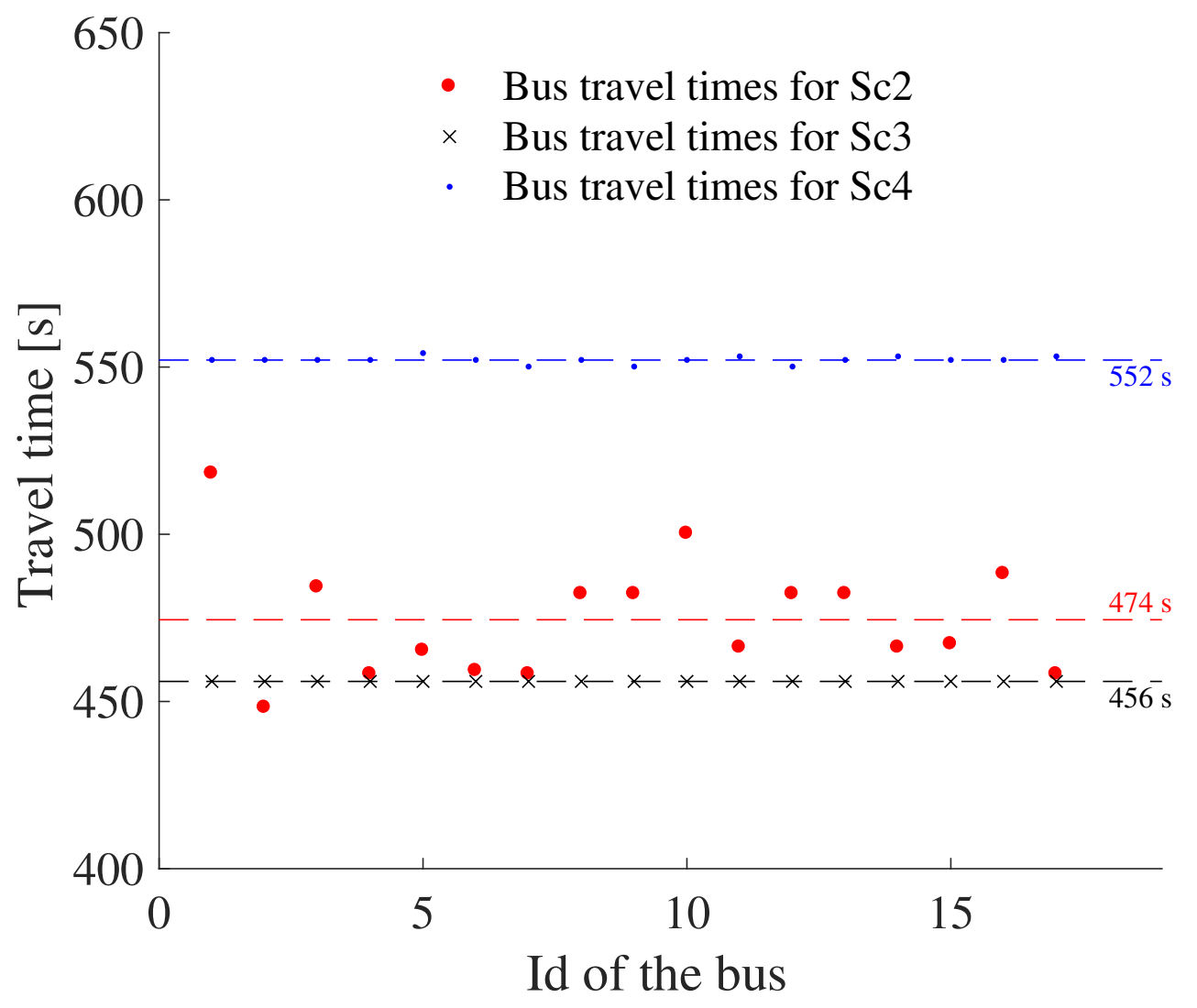

FIGURE 3 Travel time of buses through the urban arterial for scenarios $S c 2, S c 3$ and $S c 4$. Every point corresponds to one bus trip.

Due to the constant dwell time and fixed traffic signal timing, the variations in travel time are relatively small for cases $S c 4$ and $S c 3$. For $S c 2$, on the other hand, shows more variations, because traffic signal timing changes from one bus trip to the following (30). However, variations remain very limited. On the whole, buses pass significantly faster through the arterial when perimeter control is in operation in contrast to the reference case $S c 4$. This comes as every signal plan at the 14 intersections is optimized according to the current traffic situation. Thanks to the controller, almost 1.5 minute per bus can be saved in average compared to the reference scenario without any traffic management. This corresponds to a reduction in bus travel time of $15 \%$ for the three kilometers long arterial. Surely this difference in favor of case $S c 2$ will grow with increasing distances traveled. Moreover the advantages of the control scheme regarding the bus operations become even more noticeable when arterial demand is increased further. Note also, that the higher inner demand during the middle section of the simulation has no visible effect to bus travel time. 
1 Even when queue length's are taken into account, the controller still ensures good traffic conditions 2 in the arterial. The option with DBL $(S c 3)$ still is the most efficient option. Buses can travel even 3 faster than in $S c 2$ as they doesn't suffer any queues at all. However, gains of DBL compared to the controller are not significant (20 seconds or $4 \%$ per bus), considering the throwbacks of DBL described before.

Concerning the impact on traffic flow, $S c 2$ must also be favored. With DBL in operation, the assigned arterial demand in the simulation can not fully be satisfied. The number of lanes for general traffic is reduced from three two two, which results in heavy congestion outside and inside of the arterial. Thus, the available capacity for general traffic in $S c 3$ is lower than in $S c 2$. It results on spillbacks at the different intersections but also upstream of the arterial. It turns out that $S c 2$ and $S c 3$ are too dissimilar to perform a detailed comparison of the traffic conditions.

However, queues lengths can anyway be analyzed for scenarion $S c 2$. Indeed, besides the advantages provided by the controller in terms of traffic performance, the common perimeter control problem of long delay caused at the perimeter intersections is proposed to be mitigated thanks to the two-level controller. Figure 4 shows the queue lengths at the arterial entries for scenario $S c 2$ is compared. Because it is not consistent to compare with the results of scenario $S c 3$ as previously explained, we propose to compare $S c 2$ with $S c 4$, the situation without control. Our intuition is that $S c 4$ gives an underestimation of the queue length of $S c 3$. Even if no control is applied in both cases, arterial is a two-lane road in $S c 3$ whereas three lanes are simulated in $S c 4$. Note that the mean queue length over the whole simulation period is plotted. 


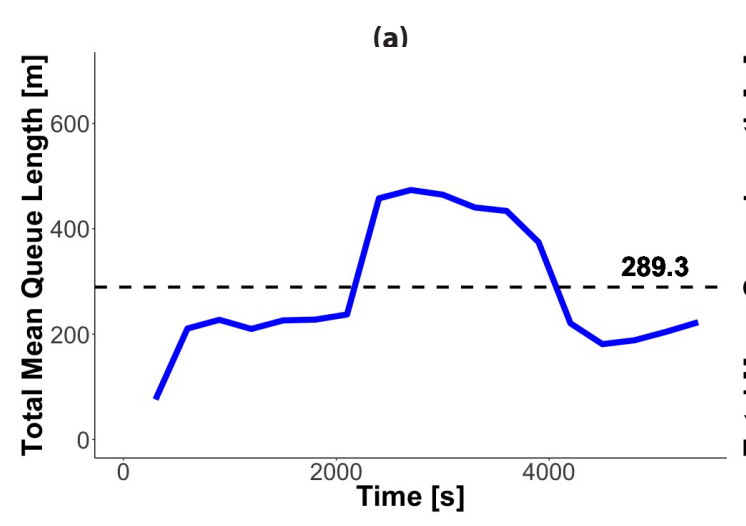

(c)

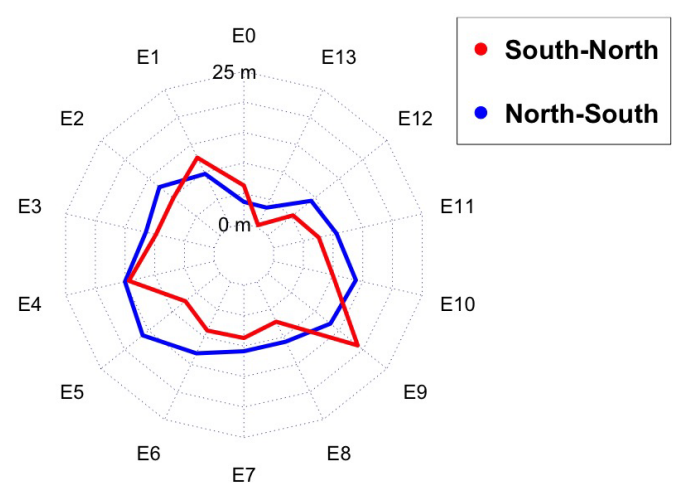

(b)

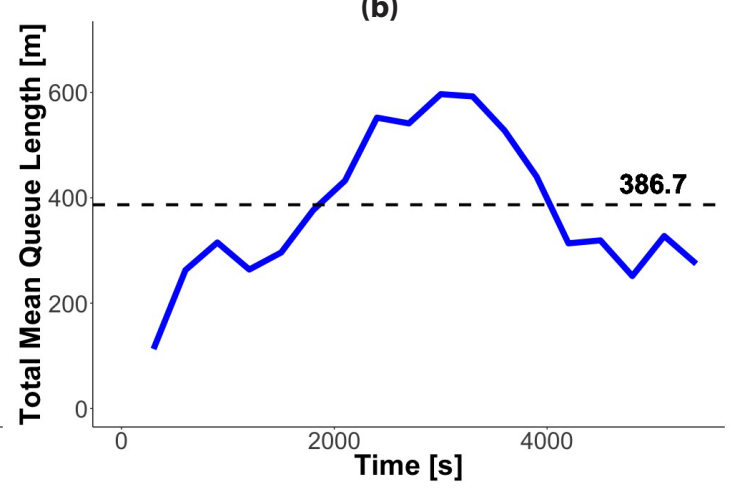

(d)

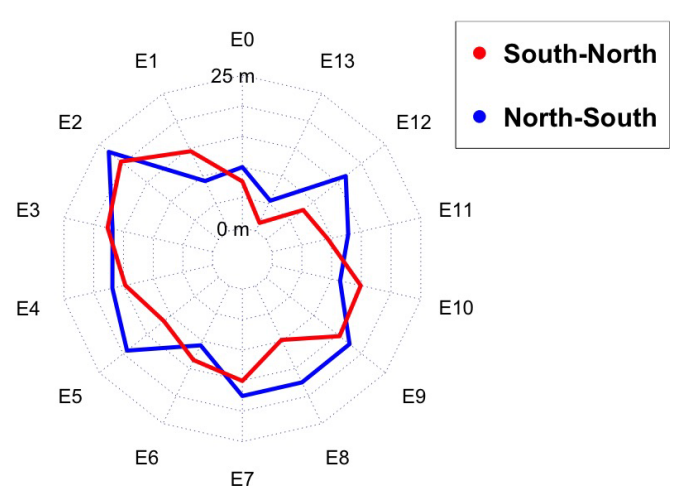

FIGURE 4 Mean queue lengths at perimeter entries cumulated over all intersections (a,b) and split up by every single intersections (c,d). Left column: $S c 4$. Right column: $S c 2$

Both diagrams for $S c 4$ and $S c 2$ show a comprehensible peak of queue lengths after half of the simulation time when inner demand from zone to zone in the network is increased. In average, the entry-queues are indeed longer when the controller is running, but the difference remains in reasonable limits and might be much smaller than in comparison to a bang-bang type of control algorithm, as pointed out by $(21)$. The kiviat (Figure $4 \mathrm{c}$ and d) charts highlight, that queues are quite evenly distributed among the intersections. Expect for entries 0 and 13, where queues are clearly below-average because these intersections are located right on the edge of the network. The equitable distribution is a consequence of a dynamic traffic assignment (DTA) applied in the simulation. Moreover, the graphs indicate, that queues on north-south approaches are somewhat longer in average than for the opposite direction. This is caused through the priority for vehicles coming from south over oncoming vehicles. Finally, because $S c 4$ gives only an underestimation of the queue length of $S c 3, S c 2$ does not have a significant effects on traffic conditions even at the intersections. These different results, i.e. decrease of bus travel times and limited effects on traffic conditions, make the replacement of DBL by perimeter control very promising.

\section{DISCUSSION AND CONCLUSION}

In this paper, we investigate, through a simulation, the potential for using perimeter control to improve bus system efficiency. This strategy aims at maintaining free-flow conditions in the vicinity of a major bus line, preventing buses from queuing together with cars at the intersections; thus, 
obtaining similar benefits to those from DBL, but without drastically reducing the capacity available for general traffic. To prevent spill-backs outside the protected area, we resort to a multi-scale perimeter controller, that is then tested with the novelty of using an urban arterial as the controlled area. The control algorithm assigns the optimal green time to each of the 14 perimeter intersections, where side streets enter into the arterial, in order to minimize total travel costs expressed as a function of vehicle accumulations. Necessary data about traffic accumulation in the arterial and queue lengths at the entering links are provided through virtual detectors. Simulation results show that traffic performance, as indicated by vehicle accumulation, mean speed and mean density in the arterial is significantly improved with the control approach applied compared to the reference case without controller.

Because traffic conditions remain in free-flow when activating the controller, it is suggested to replace dedicated bus lanes in the arterial with the proposed control scheme. As explained before, disadvantages of DBL could be eliminated thanks to this measure. The hypothesis was confirmed by simulation, which shows that perimeter control is a possible alternative to DBL. As a matter of fact, bus travel times with the perimeter control algorithm, are almost as fast as bus travel times when using a DBL. At the same time, the available capacity for general traffic is higher, and this leads to reductions in overall delays. As a result, perimeter control leads to better traffic conditions both inside and outside of the protected area. Overcoming the various drawbacks of DBL into consideration, perimeter control can therefore be a promising alternative as a traffic management strategy in urban arterials.

To further pursue this research direction, the controller can still be modified and extended. For instance, the two objective criteria for the proposed controller are weighted the same in the present study. The weighting could be adapted for other policy goals. Focus can be directed more towards queue lengths for instance. Additionally, other data sources could be used for the measurements, besides loop detectors. This could lead to an increase in the accuracy of the estimations, as these alternative technologies become more reliable, especially for high demand situations, during which loop detectors are possibly covered by vehicles and therefore, imprecise. Furthermore, the introduced feedback-controller could be extended to an MPC-model, including also a prediction of traffic conditions. Another additional option is to shorten the control period down to one or two minutes instead of five minutes, and maybe increase the efficiency of the controller.

Further comparisons could be made taking transit signal priority together with DBL into consideration, which probably results in additional time savings for buses traveling on DBL. As well, another strategy applicable are bus lanes with intermittent priority, as they represent a dynamic solution that offer more capacity to general traffic compared to DBL. In the same vein, it could be investigated if bus lanes could also been replaced when the controlled region is larger and includes a city center. Finally, the fact, that the simulated environment is simplified should be taken into consideration when results are interpreted. On a larger scale, the enhanced traffic performance through perimeter control increases the attractiveness for motorized individual traffic which results in higher demand and doesn't necessarily reduce congestion in a long term view as described by (31). Altogether, the proposed perimeter control scheme is very beneficial, because it improves the traffic conditions in a certain area and prevents it from exceeding demand.

\section{ACKNOWLEDGMENTS}

This research is partly supported by the European Research Council (ERC) under the European Unions Horizon 2020 research and innovation program (grant agreement No 646592 MAGnUM 
project). The authors thank the IT team of the traffic engineering laboratory in Licit Lyon-France for the assistance with the traffic simulator SymuVia in particular. Sincere gratitude is also expressed to the $S V T$-group in Zurich-Switzerland for providing us with the preliminary work.

\section{References}

[1] Buhaug, H. and H. Urdal, An urbanization bomb? Population growth and social disorder in cities. Global Environmental Change, Vol. 23, No. 1, 2013, pp. 1 - 10.

[2] Dargay, J., D. Gately, and M. Sommer, Vehicle Ownership and Income Growth, Worldwide: 1960-2030. The Energy Journal, Vol. 28, No. 4, 2007, pp. 143-170.

[3] Keyvan-Ekbatani, M., M. Papageorgiou, and I. Papamichail, Perimeter Traffic Control via Remote Feedback Gating. Procedia - Social and Behavioral Sciences, Vol. 111, 2014, pp. $645-653$.

[4] Rietveld, P., F. Bruinsma, and D. van Vuuren, Coping with unreliability in public transport chains: A case study for Netherlands. Transportation Research Part A: Policy and Practice, Vol. 35, No. 6, 2001, pp. 539 - 559.

[5] Amirgholy, M., M. Shahabi, and H. O. Gao, Optimal design of sustainable transit systems in congested urban networks: A macroscopic approach. Transportation Research Part E: Logistics and Transportation Review, Vol. 103, 2017, pp. 261 - 285.

[6] Balke, K., C. Dudek, and T. U. II, Development and Evaluation of Intelligent Bus Priority Concept. Transportation Research Record: Journal of the Transportation Research Board, Vol. 1727, 2000, pp. 12-19.

[7] Zhu, H., Numerical study of urban traffic flow with dedicated bus lane and intermittent bus lane. Physica A: Statistical Mechanics and its Applications, Vol. 389, No. 16, 2010, pp. 3134 $-3139$.

[8] Chiabaut, N., X. Xie, and L. Leclercq, Road Capacity and Travel Times with Bus Lanes and Intermittent Priority Activation. Transportation Research Record: Journal of the Transportation Research Board, Vol. 2315, 2012, pp. 182-190.

[9] Eichler, M. and C. F. Daganzo, Bus lanes with intermittent priority: Strategy formulae and an evaluation. Transportation Research Part B: Methodological, Vol. 40, No. 9, 2006, pp. 731 744.

[10] Chiabaut, N., Evaluation of a multimodal urban arterial: The passenger macroscopic fundamental diagram. Transportation Research Part B: Methodological, Vol. 81, 2015, pp. 410 420, optimization of Urban Transportation Service Networks.

[11] Guler, S. I. and M. Menendez, Analytical formulation and empirical evaluation of pre-signals for bus priority. Transportation Research Part B: Methodological, Vol. 64, 2014, pp. 41 - 53.

[12] Viegas, J. and B. Lu, Widening the scope for bus priority with intermittent bus lanes. Transportation Planning and Technology, Vol. 24, No. 2, 2001, pp. 87-110.

[13] Bretherton, D., G. Bowen, and K. Wood, Effective urban traffic management and control: recent developments in SCOOT. In Transportation Research Board 82nd Annual Meeting, 2003.

[14] Aboudolas, K. and N. Geroliminis, Perimeter and boundary flow control in multi-reservoir heterogeneous networks. Transportation Research Part B: Methodological, Vol. 55, 2013, pp. $265-281$.

[15] Daganzo, C. F., Urban gridlock: Macroscopic modeling and mitigation approaches. Transportation Research Part B: Methodological, Vol. 41, No. 1, 2007, pp. 49 - 62. 
[16] Keyvan-Ekbatani, M., A. Kouvelas, I. Papamichail, and M. Papageorgiou, Exploiting the fundamental diagram of urban networks for feedback-based gating. Transportation Research Part B: Methodological, Vol. 46, No. 10, 2012, pp. 1393 - 1403.

[17] Geroliminis, N., J. Haddad, and M. Ramezani, Optimal Perimeter Control for Two Urban Regions With Macroscopic Fundamental Diagrams: A Model Predictive Approach. IEEE Transactions on Intelligent Transportation Systems, Vol. 14, No. 1, 2013, pp. 348-359.

[18] Haddad, J. and A. Shraiber, Robust perimeter control design for an urban region. Transportation Research Part B: Methodological, Vol. 68, 2014, pp. 315 - 332.

[19] Ortigosa, J., M. Menendez, and H. Tapia, Study on the number and location of measurement points for an MFD perimeter control scheme: a case study of Zurich. EURO Journal on Transportation and Logistics, Vol. 3, No. 3, 2014, pp. 245-266.

[20] Haddad, J., Optimal perimeter control synthesis for two urban regions with aggregate boundary queue dynamics. Transportation Research Part B: Methodological, Vol. 96, 2017, pp. 1 $-25$.

[21] Yang, K., N. Zheng, and M. Menendez, Two-level Perimeter Control Approach in a Connected-Vehicle Environment. arXiv preprint arXiv:1612.05910, 2016.

[22] Lighthill, M. J. and G. B. Whitham, On kinematic waves. II. A theory of traffic flow on long crowded roads. In Proceedings of the Royal Society of London A: Mathematical, Physical and Engineering Sciences, The Royal Society, 1955, Vol. 229, pp. 317-345.

[23] Richards, P. I., Shock waves on the highway. Operations research, Vol. 4, No. 1, 1956, pp. 42-51.

[24] Leclercq, L., J. A. Laval, and E. Chevallier, The Lagrangian coordinates and what it means for first order traffic flow models. In Transportation and Traffic Theory 2007. Papers Selected for Presentation at ISTTT17, 2007.

[25] Xue, Z., N. Chiabaut, and L. Leclercq, Effect of Traffic Modeling on Control of Traffic Networks. Transportation Research Record: Journal of the Transportation Research Board, Vol. 2560, 2016, pp. 47-56.

[26] Herrera, J. C., D. B. Work, R. Herring, X. J. Ban, Q. Jacobson, and A. M. Bayen, Evaluation of traffic data obtained via GPS-enabled mobile phones: The Mobile Century field experiment. Transportation Research Part C: Emerging Technologies, Vol. 18, No. 4, 2010, pp. 568 $-583$.

[27] Leduc, G., Traffic Data: Collection Methods and Applications. European Commission, Joint Research Centre, Institute for Prospective Technological Studies, 2008.

[28] Daganzo, C. F. and N. Geroliminis, An analytical approximation for the macroscopic fundamental diagram of urban traffic. Transportation Research Part B: Methodological, Vol. 42, No. 9, 2008, pp. $771-781$.

[29] Basso, L. J., C. A. Guevara, A. Gschwender, and M. Fuster, Congestion pricing, transit subsidies and dedicated bus lanes: Efficient and practical solutions to congestion. Transport Policy, Vol. 18, No. 5, 2011, pp. 676 - 684.

[30] Hans, E., N. Chiabaut, and L. Leclercq, Clustering Approach for Assessing the Travel Time Variability of Arterials. Transportation Research Record: Journal of the Transportation Research Board, Vol. 2422, 2014, pp. 42-49.

[31] Duranton, G. and M. A. Turner, The Fundamental Law of Road Congestion: Evidence from US Cities. American Economic Review, Vol. 101, No. 6, 2011, pp. 2616-52. 